\title{
Probing Physics beyond the Standard Model:
}

A Search for Lepton-Flavour-Violating $Z \rightarrow \ell \tau$ Decays with the ATLAS Experiment

\author{
Ann-Kathrin Perrevoort ${ }^{a, *}$, on behalf of the ATLAS Collaboration \\ ${ }^{a}$ Radboud University and Nikhef, \\ Heyendaalseweg 135, Nijmegen, The Netherlands \\ E-mail: ann-kathrin.perrevoort@cern.ch
}

In the Standard Model, lepton flavour is conserved in all interactions. Hence, any observation of lepton flavour violation would be an unambiguous sign of physics beyond the Standard Model as predicted in numerous extensions.

One way to search for lepton flavour violation is in the decay of gauge bosons. In the search presented here, the decay of the $Z$ boson to an electron-tau or muon-tau pair is investigated using the full Run 2 proton-proton collision data set at a centre-of-mass energy of $13 \mathrm{TeV}$ recorded by the ATLAS experiment at the Large Hadron Collider. The analysis exploits tau decays into leptons and is combined with a similar analysis on hadronically decaying tau leptons.

A key ingredient to the search is the usage of a neural net to differentiate between signal and background events in order to make optimum use of the data. Combined with about 8 billion $Z$ decays recorded by ATLAS in Run 2, the strongest constraints to date are set with $\mathcal{B}(Z \rightarrow e \tau)<$ $5.0 \times 10^{-6}$ and $\mathcal{B}(Z \rightarrow \mu \tau)<6.5 \times 10^{-6}$ at $95 \%$ confidence level-finally superseding the limits set by the OPAL and DELPHI experiments more than two decades ago.

The Ninth Annual Conference on Large Hadron Collider Physics - LHCP2021

7-12 June 2021

Online

${ }^{*}$ Speaker 


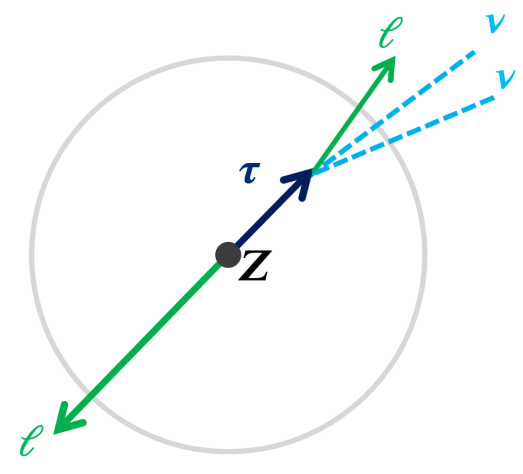

(a) The signal decay $Z \rightarrow \ell \tau$.

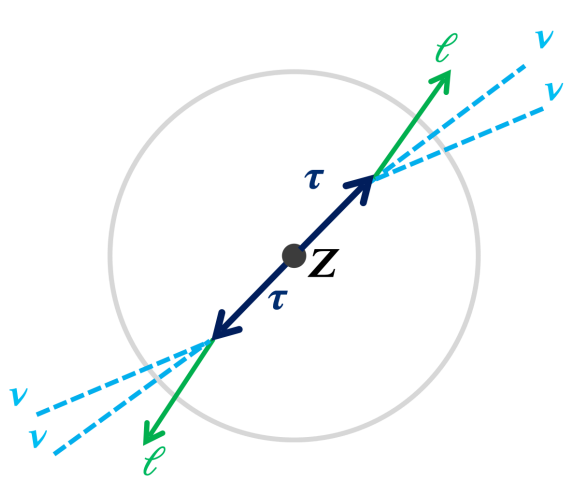

(b) The background decay $Z \rightarrow \tau \tau$.

Figure 1: Topology of (a) $Z \rightarrow \ell \tau$ signal and (b) $Z \rightarrow \tau \tau$ background events with $\tau \rightarrow \ell v \bar{v}$ decays as seen in the plane transverse to the beam direction. Figures taken from [4].

\section{Lepton Flavour Violation}

In the Standard Model of particle physics (SM), particles of each lepton family are assigned a lepton flavour quantum number which is conserved in all interactions. This conservation is however only an accidental symmetry of the model. Lepton flavour violating processes (LFV) occur in many extensions of the SM, for example in models with heavy neutrinos [1]. Moreover, the observation of neutrino oscillations indicates that LFV occurs in nature-at least in the neutrino sector. LFV processes involving charged leptons can be mediated via neutrino mixing but with far too low rates to be detected in current experiments. Thus, any observation of the LFV in charged-lepton interactions would be an unambiguous sign of physics beyond the SM.

\section{Search for $Z \rightarrow \ell \tau$ Decays}

In the ATLAS experiment [2] at the Large Hadron Collider (LHC) [3] at CERN, a search for the LFV decays $Z \rightarrow e \tau$ and $Z \rightarrow \mu \tau$ is performed analyzing proton-proton ( $p p$ ) collision data corresponding to an integrated luminosity of $139 \mathrm{fb}^{-1}$ at a centre-of-mass energy of $\sqrt{s}=13 \mathrm{TeV}$ [4]. The search exploits decays of the $\tau$ lepton into lighter charged leptons and is combined with a similar search using $\tau$ lepton decays into hadrons performed by the ATLAS experiment with $139 \mathrm{fb}^{-1}$ of $p p$ collision data at $\sqrt{s}=13 \mathrm{TeV}$ and $20.3 \mathrm{fb}^{-1}$ at $\sqrt{s}=8 \mathrm{TeV}$ [5].

The final state of the $Z \rightarrow \ell \tau \rightarrow \ell \ell^{\prime} v \bar{v}$ signal is characterized by two light, oppositely charged leptons $\ell$ and $\ell^{\prime}$ and missing energy carried by the undetected neutrinos. The invariant mass of these four particles is compatible with the mass of the $Z$ boson. The $\tau$ lepton is typically boosted in this decay due to the sizeable mass difference between the $\tau$ lepton and the $Z$ boson. Thus, the neutrinos are almost collinear with the charged lepton from the $\tau$ decay. The two charged leptons are emitted approximately back-to-back in the plane transverse to the beam direction. The topology of the signal decay is illustrated in Figure 1a.

The events are recorded with single-electron and -muon triggers. Only electron-muon pairs are considered in this analysis due to the high number of background events from $Z \rightarrow \ell \ell$ decays 
Table 1: Observed and expected (median) upper limits on the signal branching fraction at 95\% CL in the OPAL [6], DELPHI [7] experiments at LEP and the ATLAS [4, 5] experiment at the LHC.

\begin{tabular}{|c|c|c|c|}
\hline \multicolumn{4}{|c|}{ Observed (expected) upper limit on $\mathcal{B}(Z \rightarrow \ell \tau)\left[\times 10^{-6}\right]$ at $95 \%$ confidence level } \\
\hline Final state & Experiment & $e \tau$ & $\mu \tau$ \\
\hline$\ell \tau_{\text {had }}$ & OPAL [6] & 9.8 & 17 \\
\hline$\ell \tau_{\text {had }}$ & DELPHI [7] & 22 & 12 \\
\hline$\ell \tau_{\text {had }}$ & ATLAS Run $1+$ Run 2 [5] & $8.1(8.1)$ & $9.5(6.1)$ \\
\hline$\ell \tau_{\ell^{\prime}}$ & ATLAS Run 2 [4] & $7.0(8.9)$ & $7.2(10)$ \\
\hline Combined $\ell \tau$ & ATLAS Run $1+$ Run $2[4]$ & $5.0(6.0)$ & $6.5(5.3)$ \\
\hline
\end{tabular}

that would occur in the case of same-flavour lepton pairs. Since the light lepton from the $Z$ decay can be expected to have a higher momentum than the one from the $\tau$ decay, events in which the electron has a larger momentum transverse to the beam direction $\left(p_{\mathrm{T}}\right)$ than the muon are assigned to the analysis of the $Z \rightarrow e \tau \rightarrow e \mu \nu \bar{v}\left(e \tau_{\mu}\right)$ channel, and events in which the muon has a higher $p_{\mathrm{T}}$ to the $Z \rightarrow \mu \tau \rightarrow \mu e v \bar{v}\left(\mu \tau_{e}\right)$ channel.

The dominant contribution to the background stems from $Z \rightarrow \tau \tau \rightarrow \ell \ell^{\prime} v \bar{v} v \bar{v}$ decays (see Figure $1 b)$. Smaller background contributions arise from events with decays of a top-antitop-quark pair $(t \bar{t})$, two gauge bosons, or a Higgs boson. With a low probability, one of the leptons in $Z \rightarrow \ell \ell$ decays can be misidentified with the wrong flavour and thus contribute to the background. These sources of background are estimated from simulation. In addition, there is background from events with fake leptons. These stem mostly from $W(\rightarrow \ell v)+$ jets events with leptons from heavy-flavour quark decays or with light-quark initiated jets being wrongly reconstructed as electrons or muons. This type of background is estimated in a data-driven approach.

The signal region (SR) selection is based on multiplicities and kinematic selections. The final discrimination of signal and background is performed with neural network classifiers. The momenta of the reconstructed electron and muon candidates, the missing transverse momentum $E_{\mathrm{T}}^{\mathrm{miss}}$, as well as kinematic variables of the $e-\mu-E_{\mathrm{T}}^{\mathrm{miss}}$ system are taken as inputs. Three individual neural networks are trained on simulated data-one each for background events from $Z \rightarrow \tau \tau, t \bar{t}$, and two gauge bosons. Their output scores $\mathrm{NN}_{i}$ are combined into a combined $N N$ output $=1-\sqrt{\frac{1}{3} \sum_{i=1}^{3}\left(1-\mathrm{NN}_{i}\right)^{2}}$ giving equal weight to each type of background.

\section{Results}

The branching fraction $\mathcal{B}(Z \rightarrow \ell \tau)$ is extracted in a simultaneous binned maximum-likelihood fit to the combined NN output distribution in the SRs, as well as the collinear mass ${ }^{1} m_{\text {coll }}(e, \mu)$ in a dedicated $Z \rightarrow \tau \tau$ control region and the overall event yield in a dedicated $t \bar{t}$ control region. The observed and best-fit distributions of the combined NN output in the SRs of the $e \tau_{\mu}$ and $\mu \tau_{e}$ channel are shown in Figure 2.

\footnotetext{
${ }^{1}$ The collinear mass is the invariant mass of the $e-\mu-2 v$ system under the assumption that the neutrino momenta equal $E_{\mathrm{T}}^{\mathrm{miss}}$ in the plane transverse to the beam direction and have the same pseudorapidity as the subleading- $p_{\mathrm{T}}$ lepton.
} 


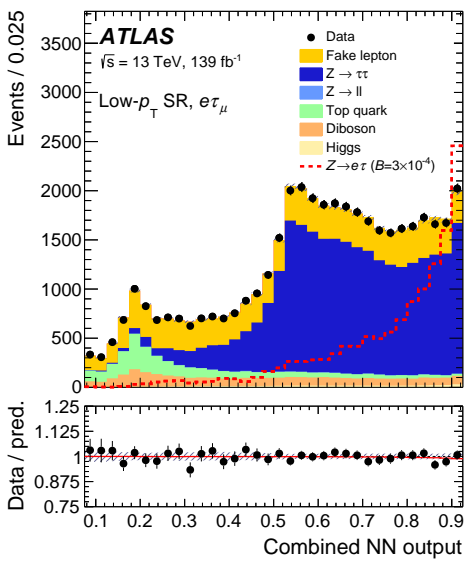

(a) Combined NN output in the $e \tau_{\mu} \mathrm{SR}$ with $p_{\mathrm{T}}(\mu)<20 \mathrm{GeV}$.



(d) Combined NN output in the $\mu \tau_{e} \mathrm{SR}$ with $p_{\mathrm{T}}(e)<25 \mathrm{GeV}$.

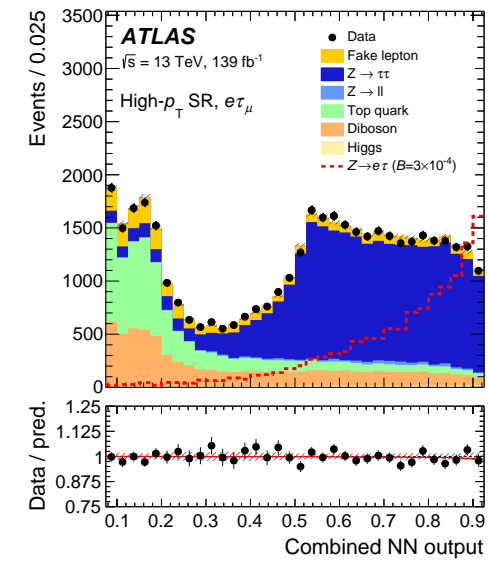

(b) Combined NN output in the $e \tau_{\mu} \mathrm{SR}$ with $p_{\mathrm{T}}(\mu)>20 \mathrm{GeV}$.

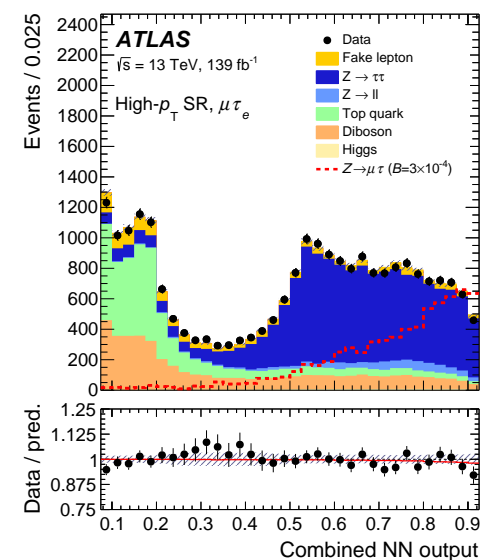

(e) Combined NN output in the $\mu \tau_{e} \mathrm{SR}$ with $p_{\mathrm{T}}(e)>25 \mathrm{GeV}$.



(c) Collinear mass in the $e \tau_{\mu}$ SR with $p_{\mathrm{T}}(\mu)>20 \mathrm{GeV}$.

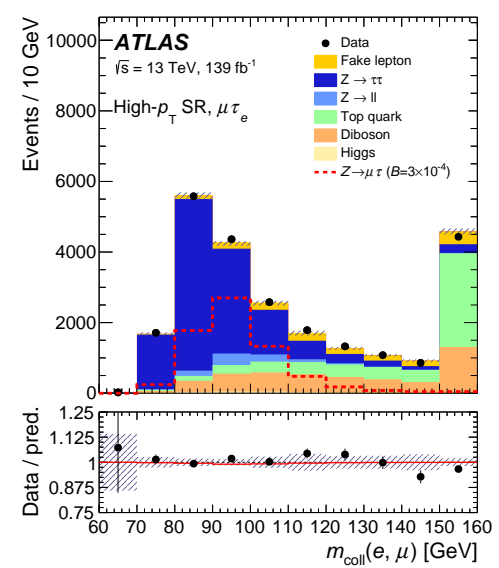

(f) Collinear mass in the $\mu \tau_{e} \mathrm{SR}$ with $p_{\mathrm{T}}(e)>25 \mathrm{GeV}$.

Figure 2: Observed and best-fit predicted distributions of the combined NN output and collinear mass in the SRs for the $e \tau_{\mu}$ and the $\mu \tau_{e}$ channel. The expected signal, normalized to an arbitrary $\mathcal{B}(Z \rightarrow \ell \tau)=3 \times 10^{-4}$, is shown as a dashed, red histogram. In the panels below each plot, the ratios of the observed yield (dots) and the best-fit background-plus-signal yield (red, solid line) to the best-fit background yield are shown. The hatched uncertainty bands represent one standard deviation of the combined statistical and systematic uncertainties. The first and last bins include underflow and overflow events, respectively. Figures taken from [4].

The analysis is combined with a similar analysis using $\tau$-lepton decays into hadrons performed by the ATLAS experiment with $139 \mathrm{fb}^{-1}$ of $p p$ collision data at $\sqrt{s}=13 \mathrm{TeV}$ and $20.3 \mathrm{fb}^{-1}$ at $\sqrt{s}=$ $8 \mathrm{TeV}$ [5]. In both cases, no excess of the data over the predicted background is observed. Observed upper limits on branching fractions are set at $\mathcal{B}(Z \rightarrow e \tau)<5.0 \times 10^{-6}$ and $\mathcal{B}<6.5 \times 10^{-6}$ at $95 \%$ confidence level. These are the strongest limits on $Z \rightarrow \ell \tau$ decays to date and supersede the limits set by the OPAL [6] and DELPHI [7] experiments at the Large Electron-Positron Collider (LEP). The observed limits are summarized in Table 1. 


\section{References}

[1] Illana, J. I. and Riemann, T., Charged lepton flavor violation from massive neutrinos in $Z$ decays, Phys.Rev.D 63053004 (2001).

[2] ATLAS Collaboration, The ATLAS Experiment at the CERN Large Hadron Collider, JINST 3 S08003 (2008).

[3] L. Evans, and P. Bryant, LHC Machine, JINST 3 S08001 (2008).

[4] ATLAS Collaboration, Search for charged-lepton-flavour violation in Z-boson decays with the ATLAS detector, submitted to Phys.Rev.Letters (2021) [hep-ex/2105.12491].

[5] ATLAS Collaboration, Charged-lepton-flavour violation at the LHC: a search for $Z \rightarrow e \tau / \mu \tau$ decays with the ATLAS detector, Nature Phys. 17 819-825 (2021) [hep-ex/2010.02566].

[6] OPAL Collaboration, A Search for lepton flavor violating $Z^{0}$ decays, Z.Phys.C-Particles and Fields 67 555-563 (1995).

[7] DELPHI Collaboration, Search for lepton flavor number violating $Z^{0}$ decays, Z.Phys.C Particles and Fields 73 243-251 (1997). 